\title{
John Ware (1807-1841) : traces et mémoire d'une trajectoire goréenne
}

\author{
Nicolas Gachon \\ Université Paul Valéry Montpellier 3
}

\section{Résumé}

Il existe peu de travaux sur les occupations britanniques de Gorée de 1663 à 1664, de 1758 à 1763, de 1779 à 1784, de 1800 à 1804 et, les Français ayant repris Gorée en 1804 mais l'ayant reperdue la même année, de 1804 à 1816. Ces périodes sont riches d'enseignements sur les relations entre les populations locales et les sujets de la puissance occupante. Elles sont en cela complémentaires aux cas mieux documentés des occupations françaises. Des unions interraciales impliquant des sujets britanniques se sont nouées à Gorée au XIX ${ }^{\mathrm{e}}$ siècle, métissages dont la mémoire parfois douloureuse, souvent parcellaire et toujours en quête de sens s'est construite pour partie autour de la conscience diffuse d'une ascendance britannique. La trajectoire du dénommé John Ware né à Gorée en 1807 sous occupation britannique et décédé en 1841 sous occupation française, la mémoire contemporaine de cette trajectoire et l'analyse de ses mécanismes forment l'objet de la présente étude de cas.

Mots clés : XIX ${ }^{\mathrm{e}}$ siècle, Sénégal, mémoire, métis, traite, engagisme.

\begin{abstract}
There are only few scholarly works on the British occupations of Gorée from 1663 to 1664, 1758 to 1763, 1779 to 1784, 1800 to 1804 and - the French having retaken Gorée in 1804 and lost it again that same year - 1804 to 1816. Those periods are quite illuminating as to the relationships between the local populations and the subjects of the occupying power. They provide perspectives that complement the better-documented cases of the French occupations. Interracial unions involving British subjects occurred in Gorée in the $19^{\text {th }}$ century; memories of a sometimes painful, often fragmented and always inquisitive nature began to form around the diffuse awareness of British ascendancies. The present case study focuses on the life-story of John Ware and aims to analyze the mechanisms underlying its contemporary memory. John Ware was born in Gorée in 1807 under British occupation. He died in 1841 under French occupation.
\end{abstract}

Keywords: 191 century, Senegal, memory, metis, slave trade, indentured trade 
Le 26 mai 1816, alors que le lieutenant-colonel James Chisholm du Royal African Corps s'apprête à quitter Gorée, les habitants lui expriment leur reconnaissance pour sa bienveillance, pour les améliorations considérables apportées sous son autorité à leur quotidien, à la défense de l'île et à l'impartialité de la justice ${ }^{1}$. Une retranscription manuscrite de ce témoignage signée par douze individus se présentant comme étant les " principaux " habitants de l'île, dont le maire Pierre Turpin, Martin Touranjou, René Dupuy, Armand Laporte, Pierre Lapolice, Nicolas Jouga et James Bradley, évoque « quelques anneaux d'or » offerts à James Chisholm « en gage de leur considération et de leur gratitude », anneaux qu'ils l'enjoignent de porter « en souvenir [d'eux] $»^{2}$. La symbolique des anneaux est féconde en ce que la strate dominante de la société goréenne marquait par là son aspiration à la pérennisation d'une forme d'union avec l'ex-occupant anglais. La plupart de ces individus, généralement français, étaient eux-mêmes engagés dans des unions «à la mode du pays » ${ }^{3}$ avec des femmes noires ou métisses certains en étaient déjà les descendants. Le cas des métissages impliquant des sujets britanniques sont ${ }^{4}$ en revanche moins connus, mais leur importance en termes de constructions mémorielles n'en est pas moindre. En cela, la confrontation entre la mémoire familiale et les traces historiques de la trajectoire de John Ware, né à Gorée en 1807 sous occupation britannique, décédé en 1841 sous occupation française, ouvre une piste de recherche intéressante.

Les ramifications généalogiques auront abouti à ce que des descendants de John Ware se trouvent aujourd'hui éparpillés dans plusieurs pays et sur plusieurs continents. Tous n'ont sans doute pas la même conscience du métissage noué sur les côtes d'Afrique de l'Ouest au début du $\mathrm{XIX}^{\mathrm{e}}$ siècle. Au Sénégal comme en Europe, les métissages renvoient à des phases historiques sensibles et font souvent l'objet de secrets perpétués en tout ou partie. Ware (ou Waar) se trouve par ailleurs être un patronyme wolof et halpulaar de la Sénégambie septentrionale, ce qui contribue à brouiller les pistes généalogiques en suscitant moins d'interrogations quant à l'éventualité d'un métissage chez les descendants africains de John Ware. Pour les descendants

\footnotetext{
${ }^{1}$ The African Institution, Eleventh Report of the Directors of the African Institution, Lxyndon, Ellerton and Henderson, 1817, p. 47.

${ }^{2}$ [Period Manuscript Copy of] « An Address from the Inhabitants of Goree to Lieut. Colonel Chisholm » [28 May 1816], Collection of the Wayfarer's Bookshop, North Vancouver, Canada.

${ }^{3}$ Les Européens qui s'installaient au Sénégal ne pouvaient généralement pas se faire accompagner de leur famille, ce qui donna lieu à un grand nombre de mariages « à la mode du pays ». Ces unions duraient habituellement le temps du séjour du mari qui, à son départ, laissait ses biens à sa concubine et à leurs enfants. De telles unions existaient depuis le $\mathrm{XV}^{\mathrm{e}}$ siècle dans les comptoirs portugais de Rufisque, de Gorée, puis de Saint-Louis ; d'où le nom de signares, du portugais Senhora, donné à ces femmes. Il s'agissait de femmes libres et parfois de niveau social enviable qui épousaient de manière élitiste des aristocrates ou des cadres négociants européens afin de tisser des réseaux d'influence. La multiplication de mariages endogamiques aboutit à l'émergence, pour reprendre la formule de Senghor, d'une sorte d'aristocratie mulâtre dont l'influence politique et économique ira grandissant au cours du XIX ${ }^{\mathrm{e}}$, lui assurant des postes clé dans les institutions, l'administration et les affaires. Voir Sylvain Sankalé, A la mode du pays. Chroniques saint-louisiennes d'Antoine François Feuiltaine, Saint-Louis du Sénégal 1788-1835, Paris, Riveneuve, 2008.

${ }^{4}$ Voir infra, note 11. Remerciements à Daniel Gachon, Paul Vernochet, Michel Pons et Magali Samat, descendants de cinquième et sixième génération de John Ware, pour leur travail de mémoire.
} 
français de cinquième et de sixième génération de la première union de John Ware recensée par le Tribunal de première instance de Saint-Louis le 8 octobre 1831, la mémoire familiale s'est en revanche construite autour de l'origine anglaise de John Ware et de ses implications probables, reconstruites ou imaginées. La mémoire familiale retient la figure d'un Anglais impliqué dans la traite atlantique qui, à Saint-Louis, aurait épousé une jeune Peule, fille d'un chef. Deux hypothèses ont été transmises à ce sujet : la première est que la jeune Peule aurait épousé John Ware pour ne pas être emportée comme esclave, la seconde que John Ware aurait épousé la fille d'un chef pour pouvoir se procurer des esclaves. Les esclaves de John Ware auraient été entreposés sur l'île de Dionewar dont le nom serait la dérivation wolofisée de " John Ware ». A Saint-Louis, la famille, sa descendance, serait originaire de N'Dar-Toute sur la Langue de Barbarie et il y aurait une trace de l'union de John Ware avec la jeune Peule dans les lettres d'Anne-Marie Javouhey ${ }^{5}$. La mémoire familiale transmet enfin une figure assez exceptionnelle, une origine peut-être noble, un pouvoir militaire ou politique, en tout cas une lignée où tous les fils aînés portaient le prénom de leur père - John.

La référence à Anne-Marie Javouhey, même si rien dans la correspondance de cette dernière n'est de nature à corroborer directement la construction mémorielle familiale, situe en revanche la trajectoire de John Ware au début des années 1820, quelque treize années après l'abolition de la traite des esclaves par les Anglais. La mémoire d'une implication de John Ware dans la traite atlantique ne pouvait se trouver totalement déconnectée d'un tel marqueur chronologique. Par ailleurs, si l'examen d'une carte du Sénégal contemporain permet de confirmer l'existence de l'île de Dionewar, son éloignement géographique de Saint-Louis soulève un certain nombre d'interrogations quant à la plausibilité d'une activité de traite atlantique impliquant John Ware depuis ces lieux. Enfin, l'hypothèse selon laquelle Dionewar serait une variante wolofisée de John Ware semble a priori fantaisiste dans la mesure où Dionewar se trouve être un nom sérère, Niominka ${ }^{6}$. Une tradition orale recueillie par Charles Becker et Victor Martin et publiée dans une collection de récits de fondations des villages rappelle en outre à quel point tradition orale et construction mémorielle peuvent s'alimenter mutuellement :

Le nom de Dionewar provient du fait qu'un navigateur portugais, nommé Dionwan, arriva au village peu après sa fondation par Ngodan [...] Celui-ci indiqua qu'il était à la recherche de son parent. Ngodan lui dit alors son nom et demanda le sien au blanc, qui lui dit qu'il s'appelait Dionwan. C'est

\footnotetext{
${ }^{5}$ Anne-Marie Javouhey, Recueil des lettres de la Vénérable Anne-Marie Javouhey, Paris, Mersch, 5 t., 1909. Fondatrice de la congrégation des sœurs de Saint-Joseph de Cluny, premier ordre de femmes missionnaires, Anne-Marie Javouhey envoya des religieuses au Sénégal en 1821, en Martinique et en Guinée en 1822, en Guadeloupe en 1823 puis en Guyane en 1828. Elle arriva personnellement au Sénégal en mai 1822.

${ }^{6}$ Le village a été fondé par des migrants Socé (Nianding) dont la langue et les pratiques religieuses sont encore aujourd'hui marquées par leur origine, le Gabou, royaume dont la plus grande partie du territoire se trouve aujourd'hui en Guinée Bissau. Remerciements à Mamadou Diouf, professeur d'histoire africaine et directeur de l'Institut d'études africaines à la Columbia University de New York.
} 
pourquoi, on donna le nom de cet étranger au nouveau village. Dionwan écrivit son nom sur un arbre du village qui a été abattu par la suite pour la construction d'une maison. ${ }^{7}$

La construction d'une mémoire familiale autour de la personne de John Ware éclaire plus largement la façon dont certains descendants de Goréens reconstruisent l'histoire de leurs ancêtres. La mémoire familiale sera ici interrogée à l'aune des traces archivistiques de la trajectoire de John Ware. Ce parcours est construit sous forme d' épisodes successifs, non consécutifs chronologiquement, qui permettent de soumettre à un examen critique les fragments de la construction mémorielle familiale : Saint-Louis, 1831 ; Gorée, 1833 ; Gorée, 1807. Suit une réflexion sur la trajectoire particulière d'un des fils de John Ware, Jacques Etienne Ware, dont la figure pour l'essentiel occultée de la mémoire familiale permet d'éclairer les mécanismes de construction mémorielle à la lumière de concepts développés par Michael Rothberg (« multidirectional memory ») ${ }^{8}$ et de Max Silverman (« palimpsestic memory »)

\section{Saint-Louis, 1831}

John Ware, commis marchand âgé de vingt-cinq ans, s'avance devant le Tribunal de première instance de Saint-Louis à l'audience du 8 octobre 1831 :

Je suis père de deux enfants naturels nés de mon union, suivant les usages du pays, avec Nebou Guiope $^{10}$, négresse libre demeurant à Saint-Louis. Ces deux enfants sont : le premier Thomas Lewis Ware né à Dagana le deux mars mil hui [sic] cent vingt-neuf, le second Jacques Etienne, également né à Dagana le trente août mil hui [sic] cent trente un. Leur naissance n'ayant pu être constatée à Dagana où il n'existe pas d'officier de l'état civil pour recevoir les déclarations, je demande à ce qu'il plaise au Tribunal de m'autoriser à faire preuve de la naissance de mes deux enfants pour cette naissance être [sic] ensuite déclarée constatée. ${ }^{11}$

Si John Ware constitue la clé de voûte de l'architecture mémorielle qui fait l'objet de la présente étude, il ne peut être l'individu autour duquel a cristallisé la mémoire véhiculée par sa descendance. Un phénomène de superposition générationnelle est ici à l'œuvre. Alors qu'il est effectivement engagé dans une union suivant les usages du pays avec une « négresse libre fûtelle même une Peule de Dagana, le fait que John Ware soit alors un simple commis marchand

\footnotetext{
${ }^{7}$ Victor Martin et Charles Becker, « Documents pour servir à l'histoire des iles du Saalum », Bulletin de l'Institut Fondamental d'Afrique Noire, t. 41, série B, nº 4, 1979, p. 722-772.

${ }^{8}$ Michael Rothberg, Multidirectional Memory: Remembering the Holocaust in the Age of Decolonization, Stanford, California, Stanford University Press, 2009.

${ }^{9}$ Max Silverman, Palimpsestic Memory: The Holocaust and Colonialism in French and Francophone Fiction and Film, NewYork, Berghahn Books, 2013.

${ }^{10}$ Nebou Guiope (désormais Nabou Diop) est vraisemblablement la transcription de Nabou Diop par des officiers français de l'état-civil. Une transcription anglaise aurait sans doute donné « Nabu Joop Rernerciements à Mamadou Diouf.

${ }^{11}$ Archives nationales d'outre-mer (ANOXA), 1DPPC4882, « Naissances constatées : Ware, Thomas Lewis ; Ware, Jacques Etienne », acte du 8 octobre 1831. Les actes de naissances sont enregistrés le 9 octobre $1831\left(\right.$ acte $^{\circ} 20$ pour Thomas Lewis Ware, né le 2 mars 1829 ; acte ${ }^{\circ} 21$ pour Jacques Etienne Ware, né le 30 août 1831).
} 
tend à invalider les hypothèses mémorielles de l'échappement à la traite pour Nabou Diop ou de l'accès à davantage d'esclaves pour lui-même. Les jeunes commis marchands travaillaient généralement pour des maisons de commerce, souvent dans des succursales où ils faisaient leurs premières armes avant de devenir marchands ou négociants à leur tour. La maison Maurel \& Prom, dont les fondateurs étaient installés à Gorée depuis 1822, disposait ainsi de succursales à Saint-Louis, Thiès, Podor, Dagana, ou encore Ziguinchor ${ }^{12}$.

Peut-on imaginer que John Ware soit africain et remettre en question son origine anglaise ? Au-delà de son propre prénom, la consonance des prénoms du premier fils reconnu lors de l'audience du 8 octobre 1831, Thomas Lewis, ne peuvent que corroborer l'hypothèse britannique. Les occupations britanniques ont été très importantes dans la reconfiguration de l'identité saint-louisienne ${ }^{13}$ et dans l'aggravation des tensions entre les « habitants » ou « originaires » (noirs libres généralement musulmans et métis) et les négociants européens ${ }^{14}$. John Ware est-il lui-même métis ? On retrouve très peu de noms à consonance britannique parmi les grandes familles métisses de Saint-Louis. La consonance des prénoms du deuxième fils reconnu lors de l'audience du 8 octobre 1831, Jacques Etienne, est de surcroit résolument francophone et chrétienne. L'identité de John Ware se trouve donc visiblement à la croisée des chemins, dans un processus d'assimilation où l'origine anglaise semble s'estomper derrière l'environnement colonial français.

Le lieu réel de la naissance des deux fils de John Ware soulève également plusieurs questions, a fortiori en lien avec une hypothèse de traite d'esclaves. Dagana se trouve à 125 kilomètres au nord de Saint-Louis, dans la basse vallée du fleuve Sénégal, dans la région historique du Waalo. Le Waalo est l'un des royaumes issus de l'éclatement de l'empire wolof au XVI ${ }^{\mathrm{e}}$ siècle. Les clans Diaw, Diop, Mbodji, Ndiaye, Ndiouck, Wade comptent parmi les plus puissants du royaume. Nabou Diop est-elle la fille d'un des chefs du Waalo ou apparentée à l'un d'entre eux ? Pour séduisante qu'elle puisse être, cette hypothèse demeure relativisée par la fréquence du nom Diop et par l'absence de documents d'état civil. L'hypothèse d'une activité de traite d'esclaves autour de Dagana ne s'en trouve pas pour autant invalidée. La traite atlantique abolie en 1807 dans le cadre britannique et en $1817-1818^{15}$ pour les commerçants français subsiste de manière clandestine, principalement dans les Rivières du Sud, entre la Gambie et la Sierra Leone, plutôt qu'au Sénégal proprement dit. L'esclavage perdure dans toutes les colonies françaises jusqu'à son abolition en 1848, abolition qui ne met fin ni à la traite interne (intra-africaine), ni à l'esclavage dans les contrées voisines de la colonie. La colonie n'a autorité que sur les territoires qu'elle administre directement pour interdire l'esclavage et la

\footnotetext{
${ }^{12}$ Remerciements à Xavier Ricou dont les recherches et communications sont particulièrement riches quant à l'histoire des métis du Sénégal.

${ }^{13}$ Saint-Louis s'est trouvée sous occupation britannique de janvier à juillet 1693, de 1758 à 1779 , et de 1809 à 1816.

${ }^{14}$ Remerciements à Mamadou Diouf.

${ }^{15}$ Il s'agissait d'une décision du Congrès de Vienne (1815) ratifiée par ordonnance royale (1817) puis par la loi (1818). Voir Serge Daget, "L'abolition de la traite des Noirs en France de 1814 à 1831 », Cahiers d'études africaines, vol. 11, nº 41, 1971, p. 14-58.
} 
traite. Victor Schœlcher lui-même s'insurge en ces termes en 1880, près d'un demi-siècle après les événements ici relatés : " à l'exception de Saint-Louis, Gorée et Dackar, des caravanes d'esclaves traversent librement des territoires français [...]. [U]n capitaine commandant la ville et le canton de Dagana prit sur lui d'arrêter un convoi ; par ordre supérieur, il dut les rendre au négrier ${ }^{16}$. Boubacar Barry dresse un constat concordant au sujet de la traite interne qui contribue « au profit des notables qui maintiennent leur position économique dominante t...] mais aussi au profit des paysans qui peuvent désormais acquérir des serviteurs domestiques. En 1904, la population servile, dans la région de Dagana, est approximativement égale en importance à la population libre ${ }^{17}$. Le contexte de la traite interne peut renvoyer à l'hypothèse d'une implication du commis marchand John Ware dans les activités potentiellement illicites de la maison de commerce pour laquelle il travaillait vraisemblablement en 1831.

Hilary Jones évoque des métissages impliquant les fondateurs de grandes maisons de commerce (Devès, Maurel \& Prom) dont les ramifications sociales sont susceptibles de pouvoir éclairer la trajectoire de John Ware ${ }^{18}$. Justin Devès était arrivé sous occupation anglaise en 1810 ; son frère Bruno avait épousé une Peule de Dagana, Ardo Ka Koumbel, avant de devenir un grand notable dont le fils était devenu maire de Saint-Louis en 1876 ; « Hubert Prom avait épousé en 1828 une riche signare, Sophie Laporte, qui lui apportait le soutien des mulâtres, et il devint président du conseil général en 1848 (son cousin Hilaire Maurel avait épousé sa bellesœur, Constance Laporte) ${ }^{19}$. Dans le sillage de ce que nous évoquions au sujet des unions à la mode du pays ${ }^{20}$, on observe ici que ces unions pouvaient revêtir une autre fonction sociale en favorisant non seulement l'intégration locale des Européens mais également leur enrichissement et, partant, leur ascension sociale voire politique. Cela étant, les tenants et aboutissants de l'union entre John Ware et Nabou Diop ne s'en trouvent que partiellement éclairés dans la mesure où ni Bruno Devès, ni Hubert Prom, ni Hilaire Maurel n'étaient de simples commis marchands. La question de l'esclavage, celle des captifs, soulève par ailleurs un certain nombre de questions liées au développement des maisons de commerce avant 1848 .

S'agissant de Maurel \& Prom, l'immeuble de la maison, longtemps le plus imposant de la ville, avait été construit par le baron $\operatorname{Roger}^{21}$. L'existence d'une captiverie au rez-de-chaussée alimente encore nombre de spéculations autour d'une implication de Maurel \& Prom dans la traite. Cette problématique repose sur une sorte de distorsion du temps et n'est donc pas de nature à éclairer notre propos. Comme le souligne Roger Pasquier, avant 1848, [1]a société

${ }^{16}$ Victor Schœlcher, L'esclavage au Sénégal en 1880, Paris, Librairie centrale des publications populaires, 1880, p. 14.

${ }^{17}$ Boubacar Barry, Le royaume du Waalo : le Sénégal avant la conquête, Paris, Karthala, 1985, p. 361.

${ }^{18}$ Hilary Jones, The Metis of Senegal: Urban Life and Politics in French West Africa, Bloomington and Indianapolis, Indiana University Press, 2013, p. 45-47.

${ }^{19}$ Jean Martin, «Les Français en Afrique subsaharienne de 1816 à 1960 », in Philippe Bonnichon, Pierre Gény et Jean Nemo (dir.), Présences françaises outre-mer (XVI $-X X I^{e}$ siècles), t. 1, Paris, Karthala, 2012, p. 505.

${ }^{20}$ Voir supra, note 3.

${ }^{21}$ Le baron Jacques-François Roger (1787-1849), gouverneur du Sénégal de 1822 à 1827, se distingua par sa politique de colonisation agricole, notamment à Dagana, et par son opposition à la traite. 
sénégalaise reposait sur le travail des esclaves qui exerçaient les métiers les plus divers : charpentiers, briquetiers, maçons, calfats, laptots ou tout simplement manœuvres $»^{22}$. La présence de ces esclaves recouvrait une réalité contrastée. Si l'abolition de la traite atlantique était devenue effective à compter de 1817-1818 pour les ressortissants français, elle n'avait pas instantanément table rase du passé : nombre d'esclaves étaient autant de séquelles de pratiques antérieures à 1848. François Zuccarelli souligne par ailleurs que le système de l'engagisme, qui, en vertu de l'arrêté du 28 septembre 1823, interdisait l'introduction de nouveaux captifs dans les possessions françaises au Sénégal tout en permettant le rachat d'esclaves pour les affranchir aussitôt, s'était en réalité développé dès $1817^{23}$. Le système dit des « engagés à temps » était censé fournir main d'œuvre et soldats tout en faisant disparaitre l'esclavage en Afrique de l'Ouest ${ }^{24}$. Cela étant, les autorités comprirent bientôt que les engagistes de Saint-Louis utilisaient de fausses déclarations pour retransformer des affranchis en esclaves. Hilaire Maurel et Hubert Prom étaient arrivés au Sénégal, à Gorée, en 1822, alors que Prom n'avait que quinze ans. Il est quasiment impossible que du commerce illicite ait gravité autour de la fameuse captiverie de Saint-Louis : il était déjà illicite à cette date de rassembler des esclaves dans une captiverie pour les réexporter, mais il n'était pas interdit de posséder des esclaves. Le bâtiment de Maurel \& Prom était essentiellement utilisé pour la gomme, principal produit traité par les marchands saint-louisiens aux escales du fleuve Sénégal dans les années 1830. Après l'ouverture d'un premier magasin par Hubert Prom à Gorée en septembre 1828, la maison Maurel \& Prom fut officiellement fondée en janvier 1831 grâce au soutien financier de son épouse. La France avait mis un terme à l'exportation d'esclaves en 1817-1818 et le Sénégal n'était plus une source d'exportation d'esclaves dans les années 1840. Les captiveries comme celle de l'édifice de Maurel \& Prom à Saint-Louis avaient été utilisées pour la traite atlantique, pas pour la traite interne. Sans doute la captiverie en question se trouvait-elle déjà dans le bâtiment au moment de son acquisition par Maurel et Prom qui, après 1848, feront bâtir de grands entrepôts sur les quais le long du fleuve pour l'échange des marchandises et des produits manufacturés importés. Dès 1750, les compagnies de commerce avaient employé les captifs des signares ou ceux des « habitants » de Saint-Louis pour les usages et métiers du quotidien, alors même qu'elles achetaient sur les marchés intérieurs des esclaves pour l'exportation qu'elles gardaient dans des captiveries sur l'île. Cette activité s'était considérablement tarie après 1790 pour s'éteindre presque totalement vers 1810 . La traite illicite s'était à ce momentlà déplacée à l'est, de la Sénégambie vers le golfe du Bénin. La consolidation de Maurel \& Prom est donc postérieure à l'interdiction de la traite, ses deux fondateurs s'étant considérablement enrichis en épousant deux jeunes femmes métisses, Sophie et Constance Laporte, filles d'Armand Laporte, maire de Gorée, négociant en gomme arabique et en arachide.

\footnotetext{
${ }^{22}$ Roger Pasquier, «A propos de l'émancipation des esclaves au Sénégal en 1848 », Revue française d'histoire d'outre-mer, t. 54, nº 194-197, 1967, p. 192.

${ }^{23}$ François Zuccarelli, «Le régime des engagés à temps au Sénégal (1817-1848)», Cahiers d'études africaines, vol. $2, \mathrm{n}^{\circ} 7,1962$, p. 428-431.

${ }^{24}$ Ibid., p. 459.
} 
Quelle donc a pu être la trajectoire qui aurait conduit John Ware d'Angleterre à SaintLouis ? L'acte de reconnaissance et les actes de naissance de Thomas Lewis et Jaques Etienne Ware ne donnent aucune indication quant à l'origine de John Ware, le qualifiant simplement de commis marchand "demeurant à Saint-Louis ${ }^{25}$. Quelle était son activité à Dagana dont l'histoire est intrinsèquement liée à celle du royaume du Waalo ? Le gouverneur Schmaltz ${ }^{26}$ avait signé le 8 mai 1819 le Traité de Ndiaw $^{27}$ avec le Brak (roi) du Waalo, Amar Faatim Borso Mbodj, et les principaux chefs : le Diawdine Madiaw Xor Aram Bakar Diaw, le Béthio Sakoura Diop, le Maalo Ndiack Danco Diaw, le Diogomaye Ndiack Arame Kélar Diaw, le Beuk Negg Ndiourbel Birame Coura Diagne. Le Traité de Ndiaw, dont le principal objectif était de permettre la colonisation agricole au Waalo, avait abouti à la création de la ville de Richard Toll en 1819 et d'une série de postes commerciaux le long du fleuve Sénégal, dont celui de Dagana en 1821. Peut-être Nabou Diop était-elle apparentée au Béthio Sakoura Diop, mais nous ne sommes pas en mesure de le vérifier. John Ware se trouvait pour sa part vraisemblablement commis au bénéfice d'une entreprise commerciale à Dagana, comptoir notamment impliqué dans le commerce de la gomme arabique, après 1821 .

La trace de John Ware se perd à Saint-Louis au-delà de 1831, date, incidemment, de la consolidation de Maurel \& Prom, ce qui nous conduit à postuler que l'audience du 8 octobre 1831 était liée à son départ imminent de Saint-Louis, et à sa volonté, même si rien n'indique qu'il ait laissé de quelconques richesses à Nabou Diop et à ses deux fils, de leur conférer à tout le moins une forme de reconnaissance sociale. S'est-il enrichi ? A-t-il acquis un statut social de plus haut rang ? Est-il retourné en Angleterre ? Est-il resté au Sénégal ?

\section{Gorée, 1833}

La trace de John Ware se retrouve deux années plus tard à Gorée sur la déclaration par « le Sieur Thomas Lewis John Ware, habitant indigène de l'Ile de Gorée, y demeurant, âgé d'environ vingt-six ans » de la naissance d'un enfant de sexe masculin ayant reçu les prénoms de François-Charles $»^{28}$. John Ware se reconnaît père de l'enfant et déclare l'avoir eu « de la Dame Henriette Turpin, aussi habitante indigène de la dite Ile de Gorée, y demeurant également avec [lui], âgée d'environ vingt-trois ans $»^{29}$. La déclaration est faite en présence « des Sieurs Arnaud Laporte, maire, âgé d'environ cinquante-neuf ans, et François de Saint-Jean, propriétaire, âgé d'environ quarante-huit ans, tous deux demeurant et domiciliés en la dite Ile de Gorée $»^{30}$. D'autres naissances de son union avec Henriette Turpin seront enregistrées par

\footnotetext{
${ }^{25}$ Voir supra, note 11.

${ }^{26}$ Le colonel Julien-Désiré Schmaltz (1771-1827), administrateur colonial français, fut gouverneur du Sénégal de 1816 à 1820 .

${ }^{27}$ Boubacar Barry, Le royaume du Waalo : le Sénégal avant la conquête, op. cit., p. 352.

${ }^{28}$ ANOM, 1DPPC4851, Naissance de François Charles-Ware », acte $n^{\circ} 8$ du 29 octobre 1833.

${ }^{29}$ Ibid.

${ }^{30}$ Ibid.
} 
l'état-civil : Rosette Amédée en $1836^{31}$, Marie Victoire en $1837^{32}$,William Théophile en $1839^{33}$. Au-delà, l'acte de décès à Joal en 1863 d'un dénommé Grégoire Ware, fils de John Ware et de Siga Diouf ${ }^{34}$, atteste l'existence d'une troisième union de John Ware.

Le nom complet de John Ware est donc Thomas Lewis John Ware, ce qui tend à corroborer la mémoire d'une lignée où tous les fils aînés portaient le prénom de leur père ${ }^{35}$. En revanche, la thèse selon laquelle John Ware serait arrivé d'Angleterre se trouve ici invalidée. John Ware est un « habitant indigène » de l'île de Gorée, un individu dont le métissage a certes pu impliquer un Anglais mais qui rend pour le moins improbable qu'il ait pu voir le jour en Angleterre. L'acte du 29 octobre 1833 ne fait plus état de sa fonction de commis marchand et ne donne aucune indication quant à sa profession. Son union avec la « Dame Henriette Turpin ", elle-même métisse et habitante indigène de l'île de Gorée, laisse entrevoir un rang social assez enviable. La famille Turpin est bien connue : Henriette est la fille de Pierre Turpin, maire de Gorée de 1817 à 1825 et l'un des douze " principaux " habitants ayant exprimé leur reconnaissance au lieutenant-colonel Chisholm le 26 mai $1816^{36}$. La personnalité des témoins conduit au même constat. Armand Laporte, maire de Gorée à la signature de l'acte, était lui aussi signataire du document adressé à James Chisholm ; il est également le père de Sophie et Constance Laporte qui avaient respectivement épousé et considérablement enrichi Hubert Prom et Hilaire Maurel ${ }^{37}$. François de Saint-Jean, lui aussi très en vue, lui aussi appelé à devenir maire de Gorée en 1849, petit-fils de Blaise Estoupan de Saint-Jean, gouverneur de Gorée de 1747 à 1758, est un personnage important dans la lignée des signares compte tenu de son union avec Caty Martin Touranjou, puis avec Anna Colas Pépin qui avait donné naissance à Mary de Saint-Jean en 1811. John Ware n'est pas un simple commis marchand. Sa trajectoire de Gorée vers Dagana et Saint-Louis avait été vraisemblablement liée au marché de la gomme, sans doute au déploiement d'une des nouvelles maisons de commerce. Maurel et Prom aussi s'étaient installés à Saint-Louis à la même période, tout en conservant leur comptoir à Gorée. Les commerçants de Gorée étaient attirés par le marché de la gomme, accessible depuis SaintLouis. Au terme de cette délocalisation momentanée, John Ware avait retrouvé une position sociale enviable à son retour en 1831, position visiblement renforcée à compter de 1838 .

En 1838, John Ware est identifié en tant que propriétaire, habitant indigène » sur l'acte notarié relatif à la vente d'un terrain sur l'Ile de Gorée. Il consent à la vente dudit terrain en sa qualité de recteur des filles mineures de feu le négociant Jean-Baptiste Baudin ${ }^{38}$ et au profit

${ }^{31}$ Ibid., « Naissance de Rosette Amédée Ware », acte $\mathrm{n}^{\circ} 10$ du 10 mars 1836.

${ }^{32}$ Ibid., « Naissance de Marie Victoire Ware 5, acte $\mathrm{n}^{\circ} 29$ du 14 juillet 1837.

${ }^{33}$ Ibid., Naissance de William Théophile Ware », acte $\mathrm{n}^{\circ} 8$ du 18 mars 1839.

${ }^{34}$ ANOM, 1DPPC5483, « Décès de Grégoire Ware », acte $\mathrm{n}^{\circ} 10 \mathrm{du} 10$ décembre 1863.

${ }^{35}$ Voir supra, note 11.

${ }^{36}$ Voir supra, note 2.

${ }^{37}$ Voir supra, note 17.

${ }^{38}$ Catherine Victoire, Sophie Rosalie et Adélaïde Marie Baudin. Leur père, Jean-Baptiste Baudin, ne doit pas être confondu avec Auguste Baudin, gouverneur du Sénégal de 1847 à 1850, qui proclama au Sénégal l'abolition de l'esclavage décrétée le 27 avril 1848 par la Deuxième République. 
d'Hubert Maurel et d'Hilaire Prom ${ }^{39}$. John Ware s'est visiblement enrichi et établi. La vente $\mathrm{du}$ terrain à Maurel et Prom vient enrichir un faisceau de concordances suggérant un lien particulier entre John Ware et Maurel \& Prom. S'il n'est fait aucune mention de sa profession sur les actes d'état-civil de Gorée après son retour de Saint-Louis, le tribunal de première instance de l'île de Gorée indique qu'il exerce " les fonctions d'huissier près le dit Tribunal » sur l'acte de naissance de son fils William Théophile le 18 mars $1839^{40}$.

L'année suivante, l'activité d'huissier de John Ware est recensée dans un extrait des minutes de l'état-civil de Gorée publié en 1842 dans un compte-rendu de la Chambre des Lords à Londres. Ce document relate une intervention de John Ware dans le processus d'affranchissement conditionnel de soixante-trois captifs débarqués à Gorée le 21 janvier 1840 par M. Marbeau, armateur de la goélette la « Sénégambie », « [d]ont acte, fait et passé en double minute au greffe de l'ile de Gorée [...] en présence des Sieurs John Ware, huissier, et François Boucher, propriétaire, tous deux demeurant en la dite île de Gorée, témoins, qui ont signé avec mon dit Sieur Marbeau et nous, Greffier, après lecture faite ${ }^{41}$. Les dérives de l'affranchissement à condition d'engagement à temps, ou engagisme, système permettant de racheter un esclave pour l'affranchir aussitôt, ont été relevées. L'affaire de la "Sénégambie » en est une. En 1839, les autorités françaises utilisent les services de l'armateur Marbeau pour recruter des engagés destinés à former une compagnie à Cayenne (Guyane). La Société Pellen et Marbeau de Saint-Louis est donc chargée du rachat de captifs aux iles Bissagos (Guinée) pour la somme de 325 francs chaque ${ }^{42}$. Cette affaire a fait l'objet d'un contentieux francobritannique car les Anglais ont saisi la «Sénégambie » et accusé les Français de se livrer à une traite déguisée sous couvert de l'engagisme ${ }^{43}$. John Ware officiera en tant qu'huissier à Gorée jusqu'à sa mort survenue à l'âge de trente-quatre ans le 11 novembre $1841^{44}$.

John Ware nait donc à Gorée sous occupation anglaise en 1807, année même où l'Angleterre abolit la traite, et meurt en 1841, sous occupation française, alors que le processus abolitionniste qui culminera en 1848 avec l'abolition de l'esclavage est déjà amorcé sous la

${ }^{39}$ Conservation des hypothèques de Saint-Louis, Vente d'un terrain situé à Gorée, par le $\mathrm{S}^{\mathrm{r}}$ Ware, au nom des mineures Baudin, aux $\mathrm{S}^{\text {rs }}$ Prom et Maurel acte $\mathrm{n}^{\circ} 32$ du 23 mai 1838. Document transmis par Xavier Ricou.

${ }^{40}$ ANOM, « Naissance de William Théophile Ware », acte $n^{\circ} 8$ du 18 mars 1839, op. cit.

${ }^{41}$ House of Lords, "Correspondence on slave trade With foreign powers parties to conventions under which vessels are to be tried by the tribunals of the nations to which they belong (From January 1, to December, 1841, inclusive) ", in The Seasonal Papers Presented by Order of the House of Lords or Presented by Royal Command in the Session $1842\left(5^{\circ} \& 6^{0}\right.$ Victoriae $)$, vol. 13, London, H.M. Stationery Office, 1842, p. 19.

${ }^{42}$ Ibid.

${ }^{43}$ François Zuccarelli, «Le régime des engagés à temps », art. cit., p. 442-443.

${ }^{44}$ ANOM, 1DPPC4852, «Décès de Thomas Levis John Ware », acte $\mathrm{n}^{\circ} 24$ du 11 novembre 1841. L'orthographe Levis est liée à la retranscription du phonème /w/. De même, le nom Ware serait parfois retranscrit sous la forme « Varre » à compter de I 'enregistrement de la naissance d'un petit-fils de John Ware, Alphonse Varre, en 1864. Certains de ses descendants portant aujourd'hui le nom Varre. Voir par exemple : ANOM, Gorée, 1DPPC4872, « Naissance de John-Théophile-Paul Varre acte $\mathrm{n}^{\circ} 90$ du 15 octobre 1890. 
Monarchie de Juillet ${ }^{45}$. Son implication dans la traite telle que transmise par la construction mémorielle est encore une fois tout à fait improbable. L'acte de décès ne recèle par ailleurs aucune information exploitable quant à l'identité, la profession et l'origine exacte de son père, sinon qu'ils sont tous deux porteurs des mêmes prénoms. Sa mère, en revanche, « Dame Rosette ${ }^{46}{ }^{4}$ (Marie-Rose) Touranjou, fille de Martin Touranjou, appartient à une lignée bien identifiée qui inscrit John Ware dans la descendance de la signare Charlotte Porquet. François Touranjou, maitre-maçon de la Compagnie des Indes, avait eu un fils, Martin, avec la signare Louise Commouille, lequel Martin avait épousé la signare Charlotte Desprez dite Porquet, demi-sœur d'Anne Pépin ${ }^{47}$, en $1778^{48}$. Dès lors, l'union de John Ware avec « Dame Henriette Turpin », les enfants recensés à compter de 1833 après son retour de Saint-Louis où il avait reconnu deux enfants d'une première union avec Nabou Diop, s'inscrit dans une lignée de souche métisse goréenne et dans un réseau social de rang privilégié.

John Ware, mulâtre, est-il véritablement d'origine anglaise ? Les sources généalogiques et autres registres d'état-civil sont de plus en plus parcellaires et finalement extrêmement rares à l'approche du début du XIX ${ }^{\mathrm{e}}$ siècle, a fortiori dans le contexte de Gorée et de ses basculements successifs entre des occupations anglaises et françaises. D'autres sources relatives à la période permettent de rassembler des faisceaux d'indices et de soulever des hypothèses. Nous avons ainsi envisagé l'hypothèse d'une origine américaine et porté une attention particulière aux travaux de l'historien américain George E. Brooks, auteur en 1970 de deux articles relatifs à l'activité d'un dénommé Enoch Richmond Ware, négociant américain actif sur les côtes d'Afrique de l'Ouest ${ }^{49}$. En l'absence de toute concordance généalogique et compte tenu du fait que le premier voyage d'Enoch Richmond Ware en Afrique a eu lieu en 1839, soit deux années avant le décès de John Ware, cette piste a pu être écartée ${ }^{50}$. En revanche, dans le cadre d'une

${ }^{45}$ Le processus était engagé depuis 1817-1818 avec l'abolition de la traite (voir supra, note 15). Dès 1834, le baron Roger, gouverneur du Sénégal de 1822 à 1827, met en garde Anne-Marie Javouhey contre les ministres qui souhaitent que les expérimentations agricoles qu'elle conduit à Mana (Guyane) n'aboutissent pas, ce qui aurait fourni de nouveaux arguments contre l'abolition de l'esclavage. Soutenue par la Société Française pour l'Abolition de l'Esclavage, Anne-Marie Javouhey parvient à réaliser l'émancipation complète d'un grand nombre d'hommes et de femmes hommes en 1838, quelques mois avant l'abolition de l'esclavage par l'Angleterre (21 août 1838). Voir Pascale Cornuel, Mère Javouhey, un cas hors normes dans la lutte contre l'esclavage », Outre-Mers, n ${ }^{\circ} 380-381,2013$, p. 63-90.

${ }^{46}$ ANOM, « Décès de Thomas Levis John Ware », acte $\mathrm{n}^{\circ} 24$ du 11 novembre 1841, op. cit.

${ }^{47}$ Anne Pépin est la sœur de Nicolas Pépin dont la maison, construite entre 1776 et 1784, est aujourd'hui communément appelée Maison des Esclaves de Gorée.

${ }^{48}$ ANOM, 1DPPC4849 « Mariage de Martin Touranjou et de Charlotte Desprez, mulâtres libres », acte $\mathrm{n}^{\mathrm{o}} 51$ du juin 1778. Voir également Jean Delcourt, Gorée. Six siècles d'histoire, Dakar, Clairafrique, 1984, p. 52.

${ }^{49}$ George E. Brooks, « Enoch Richmond Ware, African Trader; 1839-1850, Years of Apprenticeship », part 1, The American Neptune, vol. 30, 1970, p. 174-186.

${ }^{50}$ Remerciements à Robert Williams, professeur d'anthropologie au St. Mary's College of Maryland ; à Donald R. Wright, professeur d'histoire à la State University of New York (SUNY) Cortland ; à George E. Brooks, interrogé via Donald Wright. 
recherche publiée dans un article intitulé « The people of the African European frontier, from the Sahil to Sierra Leone ${ }^{51}$, Victoria Bomba Coifman a travaillé sur une lettre adressée par Robert Hughes à la Protestant Church Missionary Society en Angleterre le 1 ${ }^{\text {er }}$ juin 1816. Luimême anglais et employé par la Church Missionary Society, Robert Hughes avait ouvert une école anglaise protestante à Gorée en 1815. Alors que l'école comptait quatre-vingt-sept élèves au début de l'année 1816, Hughes en adressa la liste à la Church Missionary Society le $1^{\text {er }}$ juin 1816. John Ware, dont l'âge n'est toutefois pas mentionné, est nommément recensé dans cette liste $^{52}$. Né en 1807, il est alors âgé de neuf ans. Au terme de seize années d'occupation anglaise quasi ininterrompue, la liste compte de nombreux noms et prénoms anglophones (Begar, Bonny, Bradley, Butcher, Campbell, Charters, Farling, Flint, Gleeson, Harvey, Heddle, Hillier, Hobble, Hughes, Humbleton, Lemford, Lloyd, Mac Donough, Mooser, Otley, Pence, Ryan, Troy, Ware, Welch, Williams, Wilson), mais également des noms à consonance francophone plus connus : Angrand, Baudin, Dupuy, Lacombe, Lapolice, Pépin, Turpin ${ }^{53}$. John Ware, âgé de neuf ans, était donc scolarisé dans une école anglaise protestante à Gorée en 1816. Si l'ascendance maternelle de John Ware est désormais établie, il en va tout autrement, de son ascendance paternelle anglaise.

\section{Gorée, 1807}

Le $1^{\text {er }}$ janvier 1800, le lieutenant-colonel John Fraser est à la tête du Fraser's Corps of Infantry avec lequel il servira en Afrique de l'Ouest jusqu'en $1804^{54}$. Il sera notamment affecté à la défense de Gorée à compter d'août 1800, Gorée qui sera reprise par les Français en janvier 1804. Après la reprise de Gorée par les Anglais en mars 1804, un détachement du Royal African Corps, nouveau nom du Fraser's Corps of Infantry, désormais commandé par le lieutenantcolonel Richard Lloyd, se voit confier la défense et la destinée de l'ile. Gorée se trouve bien sous contrôle britannique à la naissance de John Ware en 1807. Le journal d'un missionnaire écossais, John Hill, parti pour l'Afrique en 1798, découvert en 1973 par Patricia Wilson, révèle la présence d'un dénommé John Ware à Gorée en 1807. David Gamble et Patricia Wilson ont notamment recensé les noms de tous les individus et les informations qui leur sont attachées dans le journal de John Hill. Il apparaît ainsi qu'un lieutenant John Ware, présent à Gorée en 1807-1808 (vraisemblablement avant 1807, mais 1807-1808 constituent les bornes chronologiques du manuscrit) a quitté l'île en septembre 1808 avec le lieutenant-colonel

\footnotetext{
${ }^{51}$ Victoria Bomba Coifman, «The People of the African-European Frontier, from the Sahil to Sierra Leone, the Rio Nunez and Rio Pongo of Lower Guinea », in G. Gaillard (dir.), Migrations anciennes et peuplement actuel des Côtes guinéennes, Paris, L'Harmattan, 2000, p. 487-515.

${ }^{52}$ Ibid., p. 492.

${ }^{53}$ Ibid., p. 492-493. L'existence et l'activité de cette école, ainsi que l'implication directe du lieutenantcolonel James Chisholm pour la soutenir sont par ailleurs évoquées dans : The African Institution, Eleventh Report, op cit., p. 126-152.

${ }^{54}$ Leslie Stephen (ed.), Dictionary of National Biography, vol. 20, London, Smith, Elder \& Co., 1889, p. 214.
} 
Richard Lloyd Lieut [john] Ware Left in Sept. 1808 with Col. Lloyd $»)^{55}$. Des recherches complémentaires au Hampshire Record Office de Winchester en Angleterre, qui détient les manuscrits de John Hill, confirment effectivement la présence du lieutenant John Ware. John Hill relate par exemple avoir visité différents lieux le jeudi 11 février 1808 accompagné du lieutenant Ware («Commander Lieut Ware »), puis avoir marché pour la première fois jusqu'aux palmiers pour admirer le paysage et avoir bu un peu de vin de palme ${ }^{56}$. La trajectoire de John Ware est donc, selon toute vraisemblance, celle d'un soldat de la couronne d'Angleterre, dont le destin semble ici lié à celui de Richard Lloyd, commandant de la garnison de Gorée. Lloyd avait servi à Gibraltar et en Jamaïque avant d'être envoyé en Afrique de l'Ouest. Il se trouve à Gorée en janvier 1801, intervient en Sierra Leone en décembre de la même année, prend formellement possession de la Gambie en novembre 1802 et est promu major en décembre 1803. Il est blessé lors des combats avec les Français en février 1804 et fait prisonnier jusqu'à leur capitulation le mois suivant.

En 1804, John Ware était devenu enseigne au régiment des York Rangers ${ }^{57}$. L'année suivante, il avait été promu lieutenant du Royal African Corps $^{58}$, en même temps qu'un dénommé Lewis Bird dont la trace se retrouve également à Gorée, dans les mêmes fonctions, dans le manuscrit de John Hill ${ }^{59}$. John Ware se trouvait donc à Gorée au moins depuis 1805. Il est identifié dans le manuscrit de John Hill en 18071808, qui fixe son retour en Angleterre, avec Richard Lloyd, au mois de septembre $1808^{60}$. On retrouve effectivement sa trace en Angleterre lorsqu'il est promu capitaine de compagnie au sein des Royal York Rangers ${ }^{61}$, nouvelle appellation du Royal African Corps à compter de 1809. Il ("Lieut. Ware, of the Royal York Rangers") épouse ensuite Ann Howard le 8 juin de cette même année à Southampton ${ }^{62}$. Il décèdera neuf ans plus tard, le 4 mars $1818^{63}$, et sera donc déjà mort au moment du baptême, le 4 avril 1818, de John Edward Ware ${ }^{64}$, seul descendant connu de son union avec Ann Howard.

\footnotetext{
${ }^{55}$ Patricia Wilson, David P. Gamble, « John Hill's Account of Life on Goree Island, 1807-1808 », in Gambia Studies Series by Professor David Gamble, $\mathrm{n}^{\circ}$ 50, Saint Mary's College of Maryland, 2006, p. 97. Remerciements à Robert Williams.

${ }^{56}$ « Thurs 11 th Feb 1808 . I went to Ducat at ${ }^{1} / 2$ past 7 after went to see Brick manufactory — about 11. The Commander Lieut Ware and I rode to the palm trees and returned about rpm. I had never been at the trees before. Thought it a very delightful and romantic scene, drank some palm wine ». Hampton Record Office (HRO), Diary of John Hill, 15M50/1574.

${ }^{57}$ The London Gazette Official Public Record, 1804, p. 330.

${ }^{58}$ Id., 1805, p. 364.

${ }^{59}$ Patricia Wilson, David P. Gamble, « John Hill's Account of Life on Goree Island », art. cit.

${ }^{60} \mathrm{Ibid}$

${ }^{61}$ The London Gazette Official Public Record, 1809, p. 912.

${ }^{62}$ The Monthly Magazine, vol. 27, $\mathrm{n}^{\mathrm{o}}$ 1, 1809, p. 632.

${ }^{63}$ The London Gazette Official Public Record, 1818, p. 474

${ }^{64}$ HRO, Southampton-St Mary, 1675-1966.
} 
L'acte de décès de Thomas Lewis John, fils mulâtre né à Gorée en 1807 de son union avec Rosette Touranjou, avait été établi en 1841 par Pierre Auguste de Saint-Just au nom des autorités françaises ${ }^{65}$. Aucun document en date de 1807, période d'occupation britannique, ne permet à ce jour d'établir sa filiation de manière absolument irréfutable. Toutefois, un faisceau de paramètres renforcé par l'improbabilité que se soient côtoyés sur l'ile de Gorée plus d'un individu d'origine britannique dénommé John Ware en 1807, rend cette hypothèse plus que probable. Le major Richard Lloyd, soldat de la couronne britannique lui aussi, a connu une trajectoire personnelle comparable à celle de John Ware durant son affectation à Gorée. C'est ainsi que disparut à Gorée, le 14 avril 1830, Marie Lloyd, fille de " Richard [Lloyd] » et de Marie Legros $^{66}$. À l'instar de Rosette Touranjou pour ce qui concerne John Ware, Marie Legros appartenait elle aussi à une lignée de signares. C'est d'ailleurs dans une maison louée à Madame Legros qu'avaient résidé Anne-Marie Javouhey et les sœurs de Saint-Joseph de Cluny en $1822^{67}$. Deux autres enfants étaient nés de l'union entre Richard Lloyd et Marie Legros : Richard en 1806, son fils aîné, porteur du prénom de son père ; et John en 1807, la même année que John Ware « fils ». Richard et John Lloyd, respectivement âgés de dix et de neuf ans, ainsi que Mary Lloyd, alors âgée de onze ans, se trouvent également recensés sur la liste d'élèves

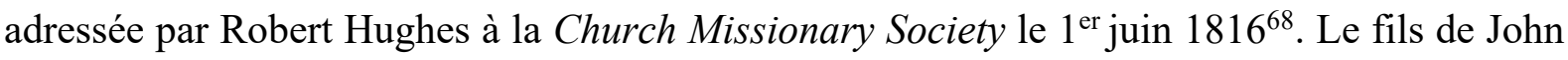
Ware et les enfants de Richard Lloyd fréquentaient donc la même école anglaise protestante en 1816 à Gorée. Lloyd et Ware avaient regagné l'Angleterre huit ans auparavant, en 1808 ; Ware devait décéder deux années plus tard, le 4 mars 1818 ; Lloyd allait être mortellement blessé, à l'âge de trente-six ans, lors de la bataille de la Nivelle, en France, le 10 décembre $1813^{69}$.

\section{Constructions mémorielles}

Un événement survenu trente-quatre ans après le décès de John Ware en 1841, quoi qu'apparemment déconnecté de la trajectoire individuelle de ce dernier, apporte un éclairage complémentaire sur le processus de construction de la mémoire familiale. Jacques Etienne Ware, l'un des deux fils de John Ware et Nabou Diop reconnus à Saint-Louis en $1831^{70}$, décède au bagne de Saint-Laurent-du-Maroni (Guyane) dans la nuit du 26 au 27 février $1879^{71}$. Curieux

\footnotetext{
${ }^{65}$ ANOM, « Décès de Thomas Levis John Ware », acte $n^{0} 24$ du 11 novembre 1841, op. cit.

${ }^{66}$ ANOM, 1DPPC4850, « Décès de Marie Lloyd, épouse du Sieur Durand Valantin », acte n ${ }^{\circ} 66$ du 14 octobre 1830.

${ }^{67}$ Anne-Marie Javouhey, Recueil des lettres de la Vénérable Anne-Marie Javouhey, op. cit., t. 2, p. 78. Sur les implications de ce séjour au Sénégal, voir Pascale Cornuel, « Mère Javouhey », art. cit.

${ }^{68}$ Victoria Bomba Coifman, « The People of the African-European Frontier », art. cit., p. 492.

${ }^{69}$ The London Gazette Official Public Record, 1813, p. 2738.

${ }^{70} \mathrm{La}$ trace de son frère aîné reste pour l'heure inconnue.

${ }^{71}$ ANOM, FRANOMCOLH404, « Ware, Jacques ». Le bagne de Saint-Laurent-sur-Maroni servait de base de transit pour des condamnés fraichement débarqués appelés à rejoindre d'autres camps et pénitenciers. Les bagnards qui restaient à Saint-Laurent étaient presque tous employés dans l'administration, souvent comme jardiniers, et étaient considérés peu dangereux et peu susceptibles de
} 
destin que le sien, condamné le 26 octobre 1875 à cinq ans de réclusion pour avoir porté des coups au dénommé Pierre Lejuge en janvier de la même année, coups portés sans intention de donner la mort mais l'ayant pourtant entrainée ${ }^{72}$. Jacques Ware avait connu un parcours chaotique et avait été déjà condamné pour des faits mineurs ${ }^{73}$. Il serait tout à fait hasardeux de gloser ici sur la question de l'absence du père, parti de Saint-Louis avant les deux ans de Jacques Etienne Ware. Cette dérive personnelle et sociale tend néanmoins à écarter l'hypothèse d'une situation confortable héritée de l'union selon les usages du pays de John Ware et de Nabou Diop. Jacques Etienne Ware avait pourtant connu des débuts honorables. En tant que soldat, d'abord, à l'instar de son grand-père britannique, puisqu'il avait fait partie du tout premier bataillon de tirailleurs sénégalais créé à l'époque de Louis Faidherbe en application du décret du 21 juillet 1857 sous Napoléon III. Il avait été décoré de la médaille militaire par décret du ministère de la Marine et des Colonies le 14 mars $1864^{74}$. Il avait épousé Virginie Barrois qui lui avait donné une fille, Élisa Catherine War[e], née à Saint-Louis le 13 avril 1859. L'acte de naissance d'Élisa War[e] précise explicitement que Jacques Ware était alors « Sergent au Bataillon de Tirailleurs Sénégalais $»^{75}$, bataillon dont le camp se trouvait alors à N'Dar-Toute sur la Langue de Barbarie, précisément à l'endroit que la mémoire des descendants retient

s'enfuir. Le dossier de bagne de Jacques Ware indique que ce dernier n'a donné lieu à aucune plainte en prison.

${ }^{72}$ Ibid.

${ }^{73}$ Condamnation le 30 août 1849 à un mois de prison pour vol (ANOM, «Ware, Jacques », op. cit.); le 20 avril 1871 à un franc d'amende pour bruit et tapage nocturne (Moniteur du Sénégal, 1871, p. 90) ; le 30 novembre 1871 à trois jours de prison pour tapage injurieux et nocturne (id., 1872, p. 114); le 22 octobre 1874 à vingt francs d'amende pour outrage à des agents de la force publique, le 19 novembre 1874 à quinze jours d'emprisonnement pour avoir tenu une maison de jeux de hasard, le 29 avril 1875 à huit mois de prison et vingt-cinq francs d'amende pour coups et blessures volontaires ANOM, «Ware, Jacques », op. cit.

${ }^{74}$ Il demandera d'ailleurs une pension de la Caisse des Offrandes Nationales au titre de cette décoration lors de son procès en 1875 , pension qui lui sera refusée. Ibid.

${ }^{75}$ ANOM, 1DPPC4898, «Elisa War, née le 13 avril 1859 », acte $\mathrm{n}^{\circ} 110$ du 14 avril 1859. L'acte de baptême révèle que sa marraine, dont la signature figure sur le document, s'appelait Catherine Ware (Paroisse de Saint-Louis, acte de baptême ${ }^{\circ} 91$ de Catherine Ware ; document transmis par Xavier Ricou). Compte tenu de son âge probable, Catherine pourrait avoir été la fille non reconnue de John Ware et de Nabou Diop, contrairement à ses deux frères Thomas Lewis et Jacques Etienne, ou avoir vu le jour après le départ John Ware de Saint-Louis. Elle épousera le dénommé François-Alphonse Bachelier, un ancien militaire devenu armurier, le 28 février 1862 à Saint-Louis (ANOM, 1DPPC4904, «Bachelier François-Alphonse marié à Ware Catherine », acte $n^{\circ} 2$ du 28 février 1862). mariage avait été annoncé en novembre 1861 dans la Feuille officielle du Sénégal (1861, p. 250). Contrairement à Thomas Lewis et Jacques Etienne Ware après la reconnaissance du 8 octobre 1831, Catherine Ware ne dispose pas d'acte de naissance au jour de son mariage. C'est sur la base d'un acte de notoriété la liant à Dame Marie-Louis Poul que le mariage sera prononcé. Dame Marie-Louis Poul comptait parmi les propriétaires qui se voyaient confier des pupilles mineurs en vertu des arrêtés des 5 décembre 1857 et 11 octobre 1862 (Moniteur du Sénégal, 1865, p. 103-104). 
comme le lieu d'origine probable de la famille. Elisa War[e] ne connaitra que très peu son père. Dès 1867, alors qu'elle n'a que huit ans, ce dernier épouse Marie Bertheloot, toujours à SaintLouis $^{76}$. Jacques Etienne Ware est désormais « guetteur au sémaphore de la barre ${ }^{77}$ après avoir été cassé du bataillon des Tirailleurs sénégalais pour inconduite et brutalité ${ }^{78}$. Elevée par sa mère Virginie Barrois, Elisa War, que ses descendants surnommeront « Mame Catho » eu égard à son second prénom, épousera en 1886 un commerçant portugais originaire de Funchal (Madère) du nom de João Fernandes Rocha ${ }^{79}$.

La trajectoire de Jacques Etienne Ware revêt une importance singulière dans le cadre de cette réflexion en ce qu'elle se trouve être absente de la mémoire familiale. En tout cas pour l'essentiel. Car si la personne de Jacques Etienne Ware semble effectivement avoir été effacée, la référence mémorielle persistante à N'Dar-Toute porte vraisemblablement sa trace. Les descendants consultés dans le cadre de cette étude, issus de l'union entre Elisa Ware et João Fernandes Rocha, et qui ont connaissance de la vie de Jacques Etienne Ware en ont une connaissance récente, liée à des recherches généalogiques. Les sources archivistiques disponibles révèlent que l'effacement était déjà perceptible au moins à compter de 1886, date du mariage d'Élisa Ware et de João Fernandes Rocha. L'acte, rédigé en présence de la mère d'Élisa War[e], stipule ainsi que la profession et le domicile de Jacques Ware sont inconnus, puis les époux attestent sous serment que le « lieu du décès » et le «dernier domicile » sont inconnus ${ }^{80}$. Élisa Ware, alors âgée de vingt-et-un ans, avait été reconnue par sa seule mère, Virginie Barois, en 1880, au cours de l'année suivant le décès de Jacques Etienne Ware ${ }^{81}$. Un effacement mémoriel allait prolonger cette sorte d'effacement administratif sans doute provoqué par la triste réputation de Jacques Étienne Ware. A la figure controversée de Jacques Etienne Ware s'est substituée la figure mémorielle de John Ware, pourtant bien moins documentée d'un point de vue archivistique : un Anglais impliqué dans la traite atlantique qui, à Saint-Louis, aurait épousé une jeune Peule, fille d'un chef. La focalisation sur les origines britanniques de John Ware avait ainsi recentré le processus mémoriel sur un plan géographique, puisque demeurait la référence à N'Dar-Toute, élément glorieux de la vie de Jacques Etienne Ware lié à sa brève carrière militaire, comme sur un plan chronologique puisque deux générations consécutives avaient été condensées en une seule, deux individus dénommés John Ware ne faisant désormais plus qu'un dans la mémoire familiale. Ce processus mémoriel prend un relief particulier à la lumière de ce que Max Silverman qualifie de palimpsestic memory, mémoire bâtie sur la «condensation » de traces spatio-temporelles différentes et parfois disparates $^{82}$. Une condensation spatio-temporelle est ici bien à l'œuvre : la mémoire familiale

\footnotetext{
${ }^{76}$ Moniteur du Sénégal, 1867, p. 554. 77.

${ }^{77}$ Ibid.

${ }^{78}$ ANOM, « Ware, Jacques », op. cit.

${ }^{79}$ ANOM, 1DPPC4943, « Joan Fernandes Rocha marié à demoiselle Élisa War, le 11 juin 1886 », acte $\mathrm{n}^{\circ} 6$ du 11 juin 1886

${ }^{80}$ Ibid.

${ }^{81}$ ANOM, 1DPPC4852, « Reconnaissance de l'enfant Elisa Ware par sa mère Virginie Barrois », acte $\mathrm{n}^{\circ} 667$ du 20 décembre 1880.

${ }^{82}$ Max Silverman, Palimpsestic Memory, op. cit., p. 10.
} 
retient l'arrivée d'un Anglais à Saint-Louis alors que c'est à Gorée, avec le Royal African Corps, que le John Ware venu d'Angleterre est réellement arrivé. Sans doute ce dernier ne s'est-il d'ailleurs jamais rendu à Saint-Louis. Les deux localisations, Gorée et Saint-Louis, ont donc été toutes les deux véhiculées par le processus mémoriel, mais elles ont été condensées et reconstruites. La mémoire familiale conserve la trace des deux localisations géographiques, des deux trajectoires, goréenne et saint-louisienne. La métaphore du palimpseste est ici féconde, celle d'un parchemin manuscrit qui aurait relaté la trajectoire de John Ware mais dont la mémoire familiale aurait effacé une partie du texte pour en écrire une autre. Il s'agit là d'un processus mémoriel éminemment productif, voire créatif, qui rappelle ce que Michael Rothberg qualifie de multidirectional memory, et qui se construit à partir de focalisations multidirectionnelles et de synergies entre des événements apparemment déconnectés dans le temps (« now and then ») et dans l'espace « here and there ») ${ }^{83}$. Le processus de construction mémorielle de la trajectoire de John Ware n'est donc ni linéaire, ni strictement conservatoire. Pour reprendre la terminologie de Max Silverman, cette mémoire crée de nouvelles configurations spatio-temporelles à partir de la recomposition d'événements distincts dans l'espace et dans le temps ${ }^{84}$.

\section{Conclusion}

Au-delà de la perspective qu'elle ouvre sur les métissages remontant aux occupations britanniques de Gorée ou de Saint-Louis, la trajectoire de John Ware et sa reconstruction dans la mémoire de ses descendants éclaire plus largement les mécanismes de la construction mémorielle. Parce que la mémoire humaine de temps aussi anciens ne peut être que parcellaire, colportée puis reconstruite, les mécanismes de reconstruction révèlent des lignes de force signifiantes. Ainsi, un phénomène de superposition s'inscrit ainsi au cœur de la dynamique mémorielle. Dans le cas de John Ware, la superposition générationnelle facilitée par l'identité des prénoms du père et du fils fusionne dans le périmètre d'une seule et même génération l'arrivée de John Ware d'Angleterre avec le Royal African Corps et l'union de son fils mulâtre avec une femme africaine de Saint-Louis. Un autre mécanisme de superposition d'ordre historique et pour une part également psychologique tient dans l'absence de distinction mémorielle entre les concepts et les pratiques de la traite locale et de la traite atlantique entre 1807 et 1841 . Peut-être faut-il y voir la reconstruction mémorielle des traces a posteriori ambiguës de pratiques engagistes auxquelles John Ware a pu participer, que ce soit en détenant lui-même des engagés (dont le statut est effectivement difficile à distinguer de celui des esclaves ${ }^{85}$ ) ou par le biais de son activité d'huissier. Il existe en effet des documents notariés relatifs à la cession de captifs signés de la main de John Ware dont, par exemple, la cession

\footnotetext{
${ }^{83}$ Michael Rothberg, Multidirectional Memory, op. cit., p. 5.

${ }^{84}$ Max Silverman, Palimpsestic Memory, op. cit., p. 3.

${ }^{85}$ Et volontairement confondus par les habitants de Saint-Louis et de Gorée. Voir supra, notes 39 et 42.
} 
d'une captive par Dame Bischop au Sieur Michel Augier en date du 16 juin 1838 pour la somme de cinq cents francs ${ }^{86}$.

La construction mémorielle est ici de nature familiale, sans mécanisme de type intégratif ou assimilatif à visée ou à portée citoyenne. Les descendants consultés sont en outre des descendants européens (français), par conséquent également nourris des problématiques mémorielles propres aux pays colonisateurs. Les métissages successifs procédant à la fusion de populations africaines de souche locale et de représentants de puissances européennes d'occupation, les mécanismes de reconstruction mémorielle ne peuvent par conséquent prétendre à la neutralité ni d'un point de vue psychologique ni d'un point de vue historique. Les écueils psychologiques sont très sensibles. La part probable de culpabilité à l'endroit de Nabou Diop, dramatisée par la rareté de sources fiables ${ }^{87}$ donnent lieu à une reconstruction de son union avec John Ware via l'épisode historiquement dramatique et de facture historique a priori très probable de l'union d'un négrier avec une femme africaine. La reconstruction de cet épisode recoupe de surcroît une tendance à l'exceptionnalisme qui se trouve être caractéristique des reconstructions mémorielles, a fortiori des reconstructions d'ordre familial. La magnification de l'image du père est un phénomène récurrent, de nature psychologique mais qui se teinte ici d'une forte exceptionnalité. La grandeur de John Ware «père » est magnifiée et dramatisée, fût-ce avec culpabilité et nonobstant le caractère sinistre mais historiquement central de son activité supposée. La même analyse doit s'appliquer à l'hypothèse mémorielle d'une wolofisation de John Ware en Dionewar, laquelle confine avec une sorte d'utopie (en l'occurrence une dystopie) mémorielle. Un autre écueil psychologique est enfin lié à la personne contrastée de Jacques Etienne Ware, condamné au bagne, dont les errements sont en partie transfigurés par le renvoi à N'Dar-Toute, camp des Tirailleurs sénégalais, donc à la respectabilité de Jacques Etienne Ware, comme site originel de la famille. La construction mémorielle s'appuie sur une sorte de mécanisme de fictionalisation qui s'applique à retracer une trajectoire déchiffrable, historiquement crédible et psychologiquement acceptable parmi les écueils historiques et psychologiques dans les interstices laissés par les éléments factuels transmis d'une génération à l'autre. Le prisme mémoriel, dès lors, est presque nécessairement ethnocentrique. Il serait par conséquent intéressant, dans le prolongement de cette recherche, de pouvoir consulter des descendants africains de John Ware, descendants issus d'autres branches et restés au Sénégal, pour procéder à une étude comparative de leurs constructions mémorielles.

\footnotetext{
${ }^{86}$ ANOM, DPPCNOTSENREP2, «Cession d'une captive par Dame Bischop au Sieur Michel Augier », acte $\mathrm{n}^{\circ} 41$ du 16 juin 1838 .

${ }^{87}$ Dont la moindre la moindre présence des femmes dans la tradition orale dans l'hypothèse où Nabou Diop serait effectivement la fille d'un chef née dans la région historique du Waalo durant les premières années du XIX ${ }^{\mathrm{e}}$ siècle.
} 


\section{Sources}

\section{Archives}

\section{Archives nationales d'outre-mer (ANOM)}

Dépôt des papiers public des colonies (DPPC)

Etat-civil, Afrique, Sénégal

Gorée :

- 1777/1824 (1DPPC4849);

- 1825-1830 (1DPPC4850);

- 1831/1840 (1DPPC4851);

- 1841/1844 (1DPPC4852);

- 1889/1890 (1DPPC4872).

Joal :

- 1861/1902 (1DPPC5483).

Saint-Louis .

- 1831/1833 (1DPPC4882);

- 1859 (1DPPC4898);

- 1862 (1DPPC4904);

- 1880 (1DPPC4930);

- 1886 (1DPPC4943).

Notariat

Gorée :

Répertoire des doubles minutes, 1831/1841 (DPPCNOTSENREP2).

Administration pénitentiaire coloniale

(Série H) Base de données des condamnés (FRANOMCOLH404).

\section{Hampshire Record Office (HRO)}

Mildmay Papers 15M50/1574.

Births \& baptisms (Parish Registers), Southampton-St Mary, 1675-1966.

\section{Périodiques et publications officielles}

Feuille officielle du Sénégal et dépendances:

- nº 99 (19 novembre 1861). 
London Gazette Official Public Record (The) :

- $\mathrm{n}^{\circ} 15684$ (17 March 1804);

- $\mathrm{n}^{\circ} 15790$ (19 March 1805);

- $\mathrm{n}^{\circ} 16268$ (20 June 1809);

- $\mathrm{n}^{\circ} 16834$ (30 December 1813);

- no 17340 (14 March 1818).

Moniteur du Sénégal et dépendances : journal officiel :

- $\mathrm{n}^{\circ} 480$ (6 juin 1865) ;

- $\mathrm{n}^{\circ} 580$ (7 mai 1867);

- $\mathrm{n}^{\circ} 791$ (9 mai 1871);

- $\mathrm{n}^{\circ} 851$ (25 juin 1872).

Monthly Magazine (The); or, British Register :

- vol. $27, \mathrm{n}^{\circ} 1,1809$.

\section{Sources imprimées}

AFRICAN INSTITUTION (THE), Eleventh Report of the Directors of the African Institution, London, Ellerton and Henderson, 1817.

HOUSE OF LORDS, "Correspondence on slave trade with foreign powers parties to conventions under which vessels are to be tried by the tribunals of the nations to which they belong (From January 1, to December, 1841, inclusive) », in The Seasonal Papers Presented by Order of the House of Lords or Presented by Royal Command in the Session $1842\left(5^{\circ}\right.$ \& $6^{\circ}$ Victoriae), vol. 13, London, H.M. Stationery Office, 1842, p. 16-78.

JAVOUHEY, Anne-Marie, Recueil des lettres de la Vénérable Anne-Marie Javouhey, Paris, Mersch, 5 vol., 1909.

SCHELCHER, Victor, L'esclavage au Sénégal en 1880, Paris, Librairie centrale des publications populaires, 1880.

STEPHEN, Leslie (ed.), Dictionary of National Biography, vol. 20, London, Smith, Elder \& Co., 1889.

\section{Articles et ouvrages historiques}

BARRY, Boubacar, Le royaume du Waalo : le Sénégal avant la conquête, Paris, Karthala, 1985.

BOMBA COIFMAN, Victoria, "The People of the African-European Frontier, from the Sahil to Sierra Leone, the Rio Nunez and Rio Pongo of Lower Guinea », in G. Gaillard (dir.), Migrations anciennes et peuplement actuel des Côtes guinéennes, Paris, L'Harmattan, 2000, p. 487-515.

BROOKS, George E., " Enoch Richmond Ware, African Trader; 1839-1850, Years of Apprenticeship », part 1, The American Neptune, vol. 30, 1970 (July), p. 174-186. 
BROOKS, George E., "Enoch Richmond Ware, African Trader; 1839-1850, Years of Apprenticeship », part 2, The American Neptune, vol. 30, 1970 (October), p. 229-248.

CORNUEL, Pascale, " Mère Javouhey, un cas hors normes dans la lutte contre l'esclavage ", Outre-Mers, ${ }^{\circ}$ 380-381, 2013-2, p. 63-90.

DAGET, Serge, «L'abolition de la traite des Noirs en France de 1814 à 1831 », Cahiers d'études africaines, vol. 11, 1971, $\mathrm{n}^{\circ}$ 41, p. 14-58.

DELCOURT, Jean, Gorée. Six siècles d'histoire, Dakar, Clairafrique, 1984.

JONES, Hilary, The Metis Of Senegal: Urban Life and Politics in French West Africa, Bloomington and Indianapolis, Indiana University Press, 2013.

MARTIN, Jean, « Les Français en Afrique subsaharienne de 1816 à 1960 », dans Philippe Bonnichon, Pierre Gény et Jean Nemo (dir.), Présences françaises outre-mer $\left(\mathrm{XVI}^{\mathrm{e}}-\mathrm{XXI}^{\mathrm{e}}\right.$ siècles), t. 1, Paris, Karthala, 2012, p. 499-606.

MARTIN, Victor et BECKER, Charles, «Documents pour servir à l'histoire des îles du Saalum », in Bulletin de l'Institut Fondamental d'Afrique Noire, tome 41, série B, n 4, 1979 (octobre), p. 722-772.

PASQUIER, Roger, " A propos de l'émancipation des esclaves au Sénégal en 1848 », Revue française d'histoire d'outre-mer, t. 54, no 194-197, 1967, p. 188208.

ROTHBERG, Michael, Multidirectional Memory: Remembering the Holocaust in the Age of Decolonization, Stanford, California, Stanford University Press, 2009.

SANKALÉ, Sylvain, A la mode du pays : Chroniques saint-louisiennes d'Antoine François Feuiltaine, Saint-Louis du Sénégal 1788-1835, Paris, Riveneuve, 2008.

SILVERMAN, Max, Palimpsestic Memory: The Holocaust and Colonialism in French and Francophone Fiction and Film, New York, Berghahn Books, 2013.

WILSON, Patricia, GAMBLE, David P., « John Hill's Account of Life on Goree Island, 1807$1808 »$ in Gambia Studies Series by Professor David Gamble, $\mathrm{n}^{\circ}$ 50, Saint Mary's College of Maryland, 2006, 110 p.

ZUCCARELLI, François, «Le régime des engagés à temps au Sénégal (18171848) », Cahiers d'études africaines, vol. 2, n 7, 1962, p. 420-461. 